\title{
Effects of Extraction, Conventional Processing and Storage on Natural Anthocyanins
}

\section{Presilski S', Presilska N ${ }^{2 *}$, Tomovska D}

${ }^{1}$ Faculty of Biotechnical Sciences, Bitola, R. Macedonia

${ }^{2}$ Metarom Australia, Food Flavouring and Colouring Industry, Sydney, Australia

*Corresponding author: Presilska N, Metarom Australia, Food Flavouring and Colouring Industry, Sydney, Australia, Tel: +61-2-9317-5002; Email: presilskan@gmail.com

Received date: December 17, 2015; Accepted date: December 28, 2015; Published date: January 07, 2016

Copyright: (c) 2016 Presilski S, et al. This is an open-access article distributed under the terms of the Creative Commons Attribution License, which permits unrestricted use, distribution, and reproduction in any medium, provided the original author and source are credited.

\begin{abstract}
The effects of extraction, conventional processing and storage on Natural Anthocyanins colorants in foods were reviewed in this project. The general knowledge available in the relevant literature is broad, yet quite not complete, especially with regards to the anthocyanins derived from food. Current knowledge does not clearly indicate if the extraction process does affect anthocyanin's properties. However, there is an indication that different extracting techniques can give different results in the quantities extracted but also in the qualitative characteristics of the extracts obtained. The conventional processing methods have deleterious effects on anthocyanins. Anthocyanin's extracts are generally not stabile under high light intensities, at high temperatures, in presence of: oxygen, metal ions, enzymes, sugars, proteins and in aqueous solutions. It would be beneficial to the industry and the consumers if a selection of techniques is made on a case basis to better suit anthocyanin preservation in manufactured products. A re-evaluation of the existing processing methods applied in processing anthocyanin containing products is recommended.
\end{abstract}

Keywords: Anthocyanins; Extraction; Processing methods

\section{Introduction}

Anthocyanins are a group of plant pigments responsible for most of the red, blue and purple hues in fruits, vegetables, cereal grains, flowers and other plant tissues in nature. Apart from their biological role as pollination attractants and phytoprotective agents, they also have economical and aesthetical role. Apparently harmless to health, anthocyanins have considerable potential in the food industry as safe and effective food additives. Not that long time ago a number of health beneficial properties of these substances have been discovered, such as: being strong antioxidants, antimutagenic and chemopreventive nutraceuticals that contribute to reduced incidences of chronic diseases and the prevention of obesity and diabetes, improvement of vision, anticancer activity [1] as well as prevention of cardiovascular diseases [2]. Hence, growing interest also lies in anthocyanins as potential nutritional supplements for humans.

\section{Anthocyanin stability as affected by extraction}

Extraction is the first step in the commercial isolation of anthocyanins. Many anthocyanin sources are known up to date. Grapes, however are the largest single fruit crop grown in the world and the most exploited one for anthocyanin extraction [3].

The extraction of anthocyanins is a delicate technological process due to their instability and the necessity to use water as an extracting solvent. The general method for commercial production involves three major steps:

(1) Maceration of the pomace in a mild solution of sulphur dioxide for 48 to $72 \mathrm{~h}$,
(2) Separation of the water phase from the spent pomace, and

(3) Vacuum concentration of the water extract, with or without recovery of a solvent.

Anthocyanin content in food products derived from fruits and berries is much smaller than the original anthocyanin content in the raw material [4].

The loss of anthocyanins in juice making is quite considerable; the receipt of blueberry anthocyanins during juice pressing was only $50 \%$. Freshly prepared cranberry juice contains a faint amount of anthocyanins $(7.7 \mathrm{mg} / 100 \mathrm{~mL})$ compared to the amount found in the berries. Also the anthocyanin content of strawberry juice is nearly half of that of strawberry pulp [3].

\section{Anthocyanin stability as affected by thermal processing}

Many foods which contain anthocyanins are thermally processed prior to consumption and this process can greatly influence anthocyanin content in the final product. Thermal processing of foods involves heating to temperatures from 50 to $150^{\circ} \mathrm{C}$, depending upon $\mathrm{pH}$ of the product and desired shelf life. Magnitude and duration of heating also has a strong influence on anthocyanin stability. The thermal degradation of anthocyanins, both in extracts and model systems, was reported to follow first-order reaction kinetics in all studies. The stability of anthocyanins and all pigments found in foods decreased with increases in temperature [5].

Heat can favour two types of discolorations associated with anthocyanins: the first one is when leucoanthocyanidins are converted into anthocyanins. This may cause pink discoloration in fruit that originally appeared cream or yellow in color. The second type involves enzymatic browning, which can be prevented by blanching and the 
addition of organic acids, such as ascorbic, malic, and citric acid [6] However, anthocyanin stability is not merely a function of the final processing temperature but is influenced by the intrinsic properties of the product and the process such as $\mathrm{pH}$, storage temperature, chemical structure and concentration of anthocyanins present, light, oxygen, the presence of enzymes, proteins and metallic ions [7].

In common with other polyphenols, anthocyanins are enzymatically degraded in the presence of polyphenol oxidase. This enzyme can be inactivated by mild heating and therefore some authors have reported that the inclusion of a blanching step (heating to approximately $50^{\circ} \mathrm{C}$ ) can have a positive effect on anthocyanin retention. For example, addition of a blanched blueberry-pulp extract to blueberry juice resulted in no degradation of anthocyanins, whereas addition of an unblanched extract caused a $50 \%$ loss of anthocyanins, suggesting an enzymatic role in anthocyanin degradation [7].

The chemical structure of anthocyanins has an effect on their resistance to degradation. In general, acylated pigments are more stable than their nonacylated counterparts. Also, anthocyanins with more glycosidic substitutions have been found to possess increased stability. In addition, higher pigment concentration and copigmentation with other phenolics present in the matrix can contribute to an increased stability of anthocyanins [6]. Several studies have shown that anthocyanins that contain several glycosylated groups acylated by aromatic acids, such as p-coumaric acid, caffeic acid, ferulic acid or sinapic acid, or by aliphatic acids (p-hydroxibenzoic, malonic, acetic, succinic, oxalic or malic) are more stable than the nonacylated equivalent [8].

Thermal degradation of anthocyanins results in the formation of polyphenolic degradation products; it is not clear if the formation of these components results in an overall reduction in antioxidant activity. This is because the polyphenolic components formed may also possess antioxidant properties, further study is required to clarify this effect especially in whole foods and not modelled systems which have been extensively studied this far [7].

Based on current knowledge it is not possible to predict the effect of thermal treatment on anthocyanin retention. The need to optimize processes in terms of quality and operating costs, demands more research to streamline processes by combinations of technologies, particularly with respect to optimisation of practical applications [7].

\section{Anthocyanin stability as affected by storage}

Anthocyanin molecules are unstable and highly susceptible to degradation during storage. The indications from most of the studies are that the kinetics of degradation of anthocyanins depends very strongly on the nature of the total system and the type of processing applied, i.e. the presence of anthocyanins, ascorbic acid and other antioxidants [9]. Degradation kinetics has been found to be of first order. However, the storage temperature and time were also found to be very influential factors responsible for anthocyanin loss during storage [10].

Cranberries stored at $20^{\circ} \mathrm{C}$ showed an anthocyanin loss in $62 \%$ of the fruit, but they exhibit a loss of only $20 \%$ when stored at $7^{\circ} \mathrm{C}$. Storage of cranberries at $0^{\circ} \mathrm{C}$ did not cause a loss of anthocyanin content, suggesting that the loss of color observed at higher temperatures might be related to enzymatic activity [6]. Another study has revealed that natural and pasteurized raspberry pulps were not very sensitive to color deterioration under processing and canning conditions, but the color practically disappeared after 50 days of storage at $37^{\circ} \mathrm{C}$. Temperature also changed the relative proportion of red raspberry anthocyanins (cyanidin and pelargonidin 3-glucosides) in juice concentrate. After 3 months of storage at $20^{\circ} \mathrm{C}$, significant changes in anthocyanins were observed while at $-20^{\circ} \mathrm{C}$, only minor changes occurred [5].

Previous studies have demonstrated that storage temperature has a decisive effect on color when these products are kept at $30^{\circ} \mathrm{C}$ for 6 months, independent on the variety or if the fruit was previously frozen or not. The resulting products have a brown hue, with no detectable free anthocyanins. Similar results were obtained when strawberry jams were analysed, indicating that when the product was stored at $37^{\circ} \mathrm{C}$, anthocyanin degradation was more than $98 \%$ after 3 months, while the same level of degradation occurred after 6 months storage at $20^{\circ} \mathrm{C}$ for all the varieties used [11]. Similarly, it has been reported that anthocyanins in Bing cherries increased slightly after canning; the authors observed a major loss in anthocyanins over 5 month storage, during which time percent polymeric colour values increased markedly in both cherry and syrup fractions. They observed an increase in percent polymeric colour values (13\% to $40 \%$ in cherries and $13 \%$ to $35 \%$ in syrup) over the 5 month storage. The authors suggested that polymeric colour could also be formed from polyphenol oxidase activity before its inhibition [7]. Anthocyanin copigmentation gives brighter, stronger, and more stable colors than those expressed by anthocyanin alone [12]. Copigmentation reactions can occur through intramolecular interactions, in which an organic acid, an aromatic acyl group, or a flavonoid (or some combination thereof) is covalently linked to an anthocyanin chromophore, or through loose intermolecular interactions, in which colorless flavonoids or other phenolic compounds [13]. The influence of light has been studied in relation to anthocyanin content and color stability of strawberry jam. The obtained results showed that no significant differences were detected between those jams stored under periodical daylight conditions and those stored in the dark for 6 months [11].

The above-mentioned results are in accordance with general findings that anthocyanin concentrations decrease during storage and that anthocyanins are markedly influenced by temperature and storage time. However the rate of color loss is slower than the rate of anthocyanin degradation. Therefore, other factors discussed elsewhere may play a significant role in the expression of color, by copigmentation, polymerization or some other physicochemical processes [11]. Although freezing is the best way to preserve the fruit aroma, it is well-known that freezing and thawing alter the flavor of fresh strawberries. In a study, five raspberry cultivars harvested at the red-ripe stage at harvest and during long-term frozen storage at $-20^{\circ} \mathrm{C}$ were evaluated. In this study the samples were rated by instrumental color analysis and by a sensory panel for color preference. The effect of freezing on instrumental hue and sensory color was not significant for any of the five varieties investigated [10]. From the above studies, it is therefore assumed that the thermal burden during processing of anthocyanic food may further degrade them during storage.

\section{Conclusion}

From the data available in literature, there is an indication that interest in anthocyanins has increased substantially over the past decades and it is expected to continue to increase. As a result, the general knowledge about these substances is broad, however, there are many aspects yet to be covered, especially with regards to the anthocyanins derived from food. Current knowledge does not indicate whether the extraction process does affect anthocyanin's properties. 
One possible reason for this gap in literature is the variety of extracting methods currently used in general industry practice. However, there is an indication that different extracting techniques can give different results in the quantities extracted but also in the qualitative characteristics of the extracts obtained. There is need for focused studies in the scope of the efficiency of different extracting methods and also in relation to their effects on anthocyanin extracts. The magnitude and duration of heating has an influence on anthocyanin stability. The stability of anthocyanins decreases with increase of temperature [5-7]. But, heat can also help inactivate oxidizing enzymes that could potentially destroy pigments present in the fruit. Therefore careful control of temperature conditions may actually benefit the color of the final product $[6,7]$. From the facts given above, it is obvious that the alternative of using some unconventional processing methods or an intelligent combination of unit operations with optimised parameters would be the most efficient way for inactivating resistant microbes and enzymes while having less impact on the anthocyanin's contents and properties. On the other hand, unconventional processing methods are performed at ambient or sublethal temperatures, therefore, the resistance of some microorganisms and enzymes, such as: polyphenol oxidase and $\beta$-glucosidase has limited their application [14-16]. Hence, it would be beneficial to the industry and the consumers if a selection of techniques is made on a case basis to better suit anthocyanin preservation in manufactured products. However, a re-evaluation of the existing processing methods applied in processing anthocyanin containing products is recommended.

\section{References}

1. Gros J (1987) Pigments in Vegetables: Chlorophylls and Carotenoids, Academic Press Inc. Ltd, London.

2. Coultate $\mathrm{T}$ (2009) Food: The Chemistry of its Components, RSC Publishing.
3. Giusti M, Jing P (2007) Natural Pigments of Berries: Functionality and Application. Berry Fruit: Value Added Products for Health Promotion, CRC Press, Boca Raton.

4. Mercadante ZA, Bobbio OF (2008) Anthocyanins in Foods: Occurence and Physicochemical Properties. Food Colorants: Chemical and Functional Properties, Taylor \& Francis, Boca Raton.

5. Gould K, Davies KM, Winefield C (2009) Anthocyanins: biosynthesis, functions and applications. Springer Science and Business Media LLC.

6. Patras A, Brunton NP, O'Donnell CP, Tiwari BK (2010) Effect of thermal processing on anthocyanin stability in foods; mechanisms and kinetics of degradation. Trends in Food Science \& Technology 21: 3-11.

7. Tiwari BK, O'Donnell CP, Cullen PJ (2009) Effect of non thermal processing technologies on the anthocyanin content of fruit juices. Trends in Food Science \& Technology 20: 137-145.

8. He J, Giusti MM (2010) Anthocyanins: natural colorants with healthpromoting properties. Annu Rev Food Sci Technol 1: 163-187.

9. Mazza G (1995) Anthocyanins in grapes and grape products. Crit Rev Food Sci Nutr 35: 341-371.

10. Francis FJ (1989) Food colorants: anthocyanins. Crit Rev Food Sci Nutr 28: 273-314.

11. Rein M (2005) Copigmentation reactions and color stability of berry anthocyanins, University of Helsinki, Finland.

12. Iversen CK (1999) Black Currant Nectar: Effect of Processing and Storage on Anthocyanin and Ascorbic Acid Content. Journal of Food Science.

13. Ancos B, Ibañez E, Reglero G, Cano MP (2000) Frozen storage effects on anthocyanins and volatile compounds of raspberry fruit. J Agric Food Chem 48: 873-879.

14. Ames JM, Hofmann TF (2000) Chemistry and Physiology of Selected Food Colorants. American Chemical society, Washington, DC.

15. Eiro MJ, Heinonen M (2002) Anthocyanin color behavior and stability during storage: effect of intermolecular copigmentation. J Agric Food Chem 50: 7461-7466.

16. Brownmiller C, Howard LR, Prior RL (2008) Processing and Storage Effects on Monomeric Anthocyanins, Percent Polymeric Color, and Antioxidant Capacity of Processed Blueberry Products. Journal of Food Science 73: 72-79. 